\title{
Gewinner und Verlierer des Aufschwungs
}

\author{
Peter Hohlfeld, Camille Logeay, Rudolf Zwiener
}

Der im Jahr 2004 begonnene Aufschwung dürfte Mitte 2008 zu Ende gegangen sein. Es war ein kräftiger und lang anhaltender Aufschwung. Nach etlichen Jahren Stagnation waren viele Hoffnungen mit ihm verbunden: dass sich für viele die Einkommens- und Beschäftigungssituation deutlich verbessern möge, dass sich die schmerzhaften Sozialstaatskürzungen - die Reformen der Agenda 2010 - auszahlen und die staatlichen Finanzen endlich saniert sein würden. Diese Hoffnungen spiegeln sich in der Aussage der Bundeskanzlerin auf dem CDU-Parteitag Ende 2007 wider, der Aufschwung komme bei immer mehr Menschen an. Wie kann es dann aber sein, dass die regelmäßig veröffentlichten Meinungsumfragen eine völlig andere Wahrnehmung der Bevölkerung offenbaren und dass immer häufiger über die Schwierigkeit vieler Haushalte, über die Runden zu kommen, berichtet wird? Gibt die mediale Welt ein verzerrtes Bild wieder? Wohl kaum. Denn wir wissen, dass Armut und Einkommensungleichheit in den letzten Jahren zugenommen haben. Und dieser Trend ist - anders als erhofft-weder umgekehrt noch aufgehalten worden. Für eine derart enttäuschende Feststellung mehren sich die Indizien.

Die Beiträge dieses Schwerpunktheftes zeichnen anhand der zahlreich präsentierten Indikatoren und Analysen ein Bild, das erkennen lässt, dass der Aufschwung nur bei wenigen ankommt. Die Zahl der Beschäftigten ist zwar gestiegen, doch der Trend zu immer mehr atypischen Beschäftigungsverhältnissen ist nicht durchbrochen worden - und dies trotz einer Zunahme der sozialversicherungspflichtigen Beschäftigung. Der Anstieg war letztlich dem vorhergehenden Aufschwung zu ähnlich. Dagegen macht sich der geringe Lohnanstieg nach über drei Jahren guter Konjunktur in den Haushaltskassen bemerkbar. Die realen Nettolöhne pro Kopf sind sogar bis zuletzt zurückgegangen. Feinere Untergliederungen - allerdings nur bis 2006 möglich - zeigen, dass sich die Einkommensverteilung insgesamt zulasten der unteren Einkommensgruppen verschoben hat. Aber auch Kerngruppen des Arbeitsmarktes sind von Niedriglöhnen betroffen und der wachsende Niedriglohnsektor entfaltet Sogwirkungen in anderen Bereichen. Dies bestätigt die öffentliche Wahrnehmung, dass die Mehrheit der Arbeitnehmer vom Aufschwung nicht profitiert hat - und schon gar nicht diejenigen, die sich im Bereich der unteren Lohnskala finden.

Verfolgt man die Entwicklung der funktionalen Einkommensverteilung bis zum aktuellen Rand, sieht man, wie ungleich sich die Einkommen im Aufschwung entwickelt haben. Obwohl die Beschäftigung zu- und die Arbeitslosigkeit abgenommen hat, steigt die gesamte Lohnsumme nicht in dem Maße, wie es in Aufschwüngen sonst üblich war. Real hat sie insgesamt gerade mal dasselbe Niveau erreicht wie zu Beginn des Aufschwungs. Hingegen sind die Gewinne geradezu explodiert.

Diese von vielen als ungerecht empfundene Entwicklung hat nicht nur politische Konsequenzen, sie führt auch zu einem besorgniserregenden Missverhältnis bei der Wachstumsstruktur; die deut- sche Wirtschaft wird sehr stark vom Außenhandel getragen und im Wesentlichen profitiert nur die Industrie davon. Das macht Deutschland immer abhängiger von der Weltkonjunktur, insbesondere von der europäischen. Auch die europäischen Partner leiden unter diesem faktischen Lohndumping Deutschlands, was das reibungslose Funktionieren der Europäischen Währungsunion schwer belastet. Es ist geradezu blanke Ironie, dass ein Teil des Lohndrucks auch durch die von europäischer Ebene initiierten Haushaltskonsolidierungen mit verursacht wurde.

Es ist Zeit, diese wirtschaftlichen Fehlentwicklungen nicht nur zu erkennen, sondern auch zu korrigieren. Mindestlöhne sind ein Weg, um Lohnungleichheiten zu reduzieren und insgesamt wieder zu Lohnerhöhungen zu kommen, die sich am Inflationsziel der Europäischen Zentralbank (EZB) und der durchschnittlichen Produktivitätsentwicklung orientieren. Um eine weitere Zunahme der Einkommensungleichheit zu verhindern und Armut erfolgreich zu bekämpfen, bedarf es allerdings weiterer Maßnahmen. So müssen die Globalisierungsgewinne gerechter verteilt werden, um die Akzeptanz für eine weitere Integration in die Weltwirtschaft zu sichern. Auf europäischer Ebene müssen sich alle aktiv für die Einhaltung der Sozialstandards einsetzen. Und nicht zuletzt kommt der Finanzpolitik eine Schlüsselrolle zu. In den letzten Jahren hat gerade die Finanzpolitik ihre Stabilisierungs- und Umverteilungsfunktion nicht mehr wahrgenommen und mit ihren diskretionären Maßnahmen die Ungleichheit sogar noch verstärkt. Offensichtlich war es nicht mehr Ziel der staatlichen Umverteilungspolitik, die Einkommensdiskrepanzen $z u$ korrigieren. Eher war das Gegenteil der Fall: Auf allen staatlichen Aktionsfeldern wurden die Weichen so gestellt, dass die disparate marktmäßige Entwicklung zugunsten der oberen Einkommensschichten verstärkt wurde. Indes obliegt es gerade dem Staat, die inzwischen aufgelaufenen Probleme, insbesondere hinsichtlich der Verteilungsproblematik, zu lösen und dafür zu sorgen, dass die Marktwirtschaft auch eine soziale ist und bleibt.

\footnotetext{
Peter Hohlfeld, Wissenschaftler im Institut für Makroökonomie und Konjunkturforschung (IMK) in der Hans-Böckler-Stiftung. Arbeitsschwerpunkte: Volkswirtschaftliche Gesamtrechnungen und Konjunkturanalyse e-mail: peter-hohlfeld@boeckler.de Dr. Camille Logeay, Wissenschaftlerin im IMK in der Hans-BöcklerStiftung. Arbeitsschwerpunkte: Makroökonomische Grundlagenforschung und Arbeitsmarkt. e-mail: camille-logeay@boeckler.de Dr. Rudolf Zwiener, Wissenschaftler im IMK in der Hans-BöcklerStiftung. Arbeitsschwerpunkte: Modellsimulationen und wirtschaftspolitische Beratung. e-mail: rudolf-zwiener@boeckler.de
} 